\title{
Measurement and Comparison of Output Factors Using Two Detectors for NOVAC7 Intra0perative Radiotherapy Accelerator
}

\author{
Md Rafiqul Islam ${ }^{1,2,3 *}$, Hiroshi Watabe1, Andreoli Stefano ${ }^{3}$ \\ ${ }^{1}$ Graduate School of Biomedical Engineering, Tohoku University, Aoba-ku, Japan \\ ${ }^{2}$ Institute of Nuclear Medical Physics, AERE, Bangladesh Atomic Energy Commission, Dhaka, Bangladesh \\ ${ }^{3}$ Medical Physics Department, ASST Papa Giovanni XXIII Hospital, Bergamo, Italy \\ Email: *mripbaec@gmail.com
}

How to cite this paper: Islam, M.R., Watabe, H. and Stefano, A. (2020) Measurement and Comparison of Output Factors Using Two Detectors for NOVAC7 IntraOperative Radiotherapy Accelerator. International Journal of Medical Physics, Clinical Engineering and Radiation Oncology, 9, 52-61.

https://doi.org/10.4236/ijmpcero.2020.92006

Received: March 13, 2020

Accepted: April 12, 2020

Published: April 15, 2020

Copyright $\odot 2020$ by author(s) and Scientific Research Publishing Inc. This work is licensed under the Creative Commons Attribution International License (CC BY 4.0).

http://creativecommons.org/licenses/by/4.0/

\begin{abstract}
The aim of this work was to evaluate and compare the performance of comparatively new synthetic PTW 60019 microDiamond with PTW 60017 Diode E detector in measuring the output factors (OF) of IntraOperative Radiation Therapy (IORT) electron beams. For a given electron beam, OFs are defined as the ratio of the dose for any applicator size at the depth of maximum to that for a reference applicator. IORT is an innovative treatment technique that delivers a large single fraction of radiation dose to the tumor bed during surgery. The electron beams considered in this study were generated by the mobile NOVAC7 system. This device produces high-dose-per-pulse electron beams with four different energies in the range from $3 \mathrm{MeV}$ to $9 \mathrm{MeV}$. We performed measurements for two higher energies, namely $7 \mathrm{MeV}$ and $9 \mathrm{MeV}$. The beam collimation was performed through Perspex (PMMA) cylindrical applicators with different diameters. The accurate dose delivery of IORT tightly depends on the precision of measured dose by reference applicator and the output factors of clinical applicators. The output factors were measured using microDiamond and Diode E detectors. The microDiamond detector performance was compared with a Diode E detector. Determined output factors of two detectors were in good agreement. The maximum deviations of output factors for microDiamond were found $2.74 \%$, and $2.17 \%$ for $7 \mathrm{MeV}$ and 9 $\mathrm{MeV}$, respectively with respect to the PTW Diode E. The microDiamond detector was shown to exhibit excellent properties for output factor measurements and could be considered as a suitable tool for electron beam dosimetry.
\end{abstract}

\section{Keywords}

IntraOperative Radiation Therapy (IORT), NOVAC7, Detectors, Output Factors 


\section{Introduction}

IntraOperative radiation Therapy (IORT) is a technique that delivers a high single fraction of radiation dose to the tumor during the surgical procedure. IORT can be combined with external beam radiation therapy (EBRT) or used as a single radiation dose [1] [2]. Recently, some types of dedicated accelerators including Mobetron, NOVAC and LIAC are introduced for IORT. NOVAC and LIAC are able to produce a high dose per pulse electron beam and possible to apply the whole dose of radiation during the surgery in the operating room. The NOVAC7 accelerator system is different from the conventional linacs firstly, because it is a mobile accelerator. Moreover, the NOVAC7 is equipped with a "hard-docking" collimation system with a design considerably different from those currently used for IORT. The characteristics of the NOVAC7 beams and those of the other IORT beams are then expected to be appreciably different from each other. Most important feature of this mobile dedicated radiotherapy device is the production of a very high dose per pulse electron beam. There are many advantages of IORT modality: sharp dose falloff, ideal dose distribution, short treatment time, high radiobiological effectiveness and normal tissue protection [3]. On the contrary, the high dose rate used by mobile accelerators can affect the accuracy of the dosimetric systems utilized for accelerator commissioning and quality assurance measurements.

The irradiation procedure in IORT is usually determined based on tabulated data which describe the dose distribution according to the energy, field size and depth. It is a duty of a medical physicist to find such beam parameters that the dose distribution fits best with prescribed dose. The dose distribution from electron beams is difficult to predict due to complex dependence on the beam energy and complicated trajectory of particles affected by scattering foils, collimating elements, such as applicator inserts and patient body. The dose per Monitor Unit (D/MU) can be calculated by the relative output factor (OF).

Ionization chambers are the most frequently used equipment in dosimetry of radiation therapy [4]. The dosimetric characterization of IORT beams is a non-trivial and time-consuming procedure, often requiring the use of different detectors. Ionization chambers can also be used to perform the dosimetric measurements at dose per pulse, but charge collection efficiency must be determined taking into account the free electron effects on the charge transport in the air cavity [5]. In order to overcome inaccuracy with ionization chambers measurements of IORT fields, several different correction methods have been proposed in recent years. So, it may be convenient to use less angular, energy and correction factor dependent such as p-type diode, synthetic diamond, Fricke gel dosimetry, electron paramagnetic resonance with Alanine. In recent, due to high spatial resolution, solid state detectors are used in IORT electron beams for dose profile measurements and relative dosimetry [6]. Recently PTW Freiburg, Germany introduced a synthetic single crystal diamond based PTW 60019 microDiamond detector with the development in Rome University "Tor Vergata" [7]. 
In particular, due to its high spatial resolution comparable to that of commercial silicon diode, superior tissue equivalence and no dose rate dependence, the PTW 60019 microDiamond detector is recommended by the manufacturer for relative dosimetry in large and small electron and photon beams [8]. Output Factor (OF) is given by the ratio of detector reading for the specific applicator to the reading of reference applicator. Some studies aimed to determine of the most adequate detectors to use under high DPP electron beams from IORT system by comparing their OF, but nobody has done such kind of study for NOVAC7 electron beams [9] [10]. The aim of this study was to introduce the new PTW 60019 microDiamond detector for output factors measurement of NOVAC7 mobile accelerator electron beams and comparing the results of those of PTW 60017 Diode $\mathrm{E}$ detector. The Diode $\mathrm{E}$ is a solid state detector and it is considered a suitable dosimeter for dose measurements in small electron fields due to its small size, small sensitive volume, high sensitivity, allowing accurate beam profile and percentage depth dose (PDD) measurements. For these reasons Diode E are well assessed in clinical relative electron dosimetry [11]. In literature, [12] G. Güngör et al., mentioned that PTW Diode E detector demonstrated convenient OFs results of IORT electron beams comparatively others solid state detectors.

\section{Material and Methods}

\subsection{IORT Accelerator}

Irradiation were performed using the 3D movable NOVAC7 (Hitesys SpA, Aprilia (LT) Italy 1997) which was installed and commissioning few years ago in the ASST Papa Giovanni XXIII Hospital in Bergamo, Italy are shown in Figure 1.

The NOVAC7 is a mobile accelerator with the dimension of $232 \mathrm{~cm}$ in length and $194 \mathrm{~cm}$ in height, specifically designed to perform intraoperative radiotherapy. This device accelerates electrons to four different nominal energies in the range from $3 \mathrm{MeV}$ to $9 \mathrm{MeV}$. These energies are denoted with the codes $\mathrm{A}_{-}(3 \mathrm{MeV})$,

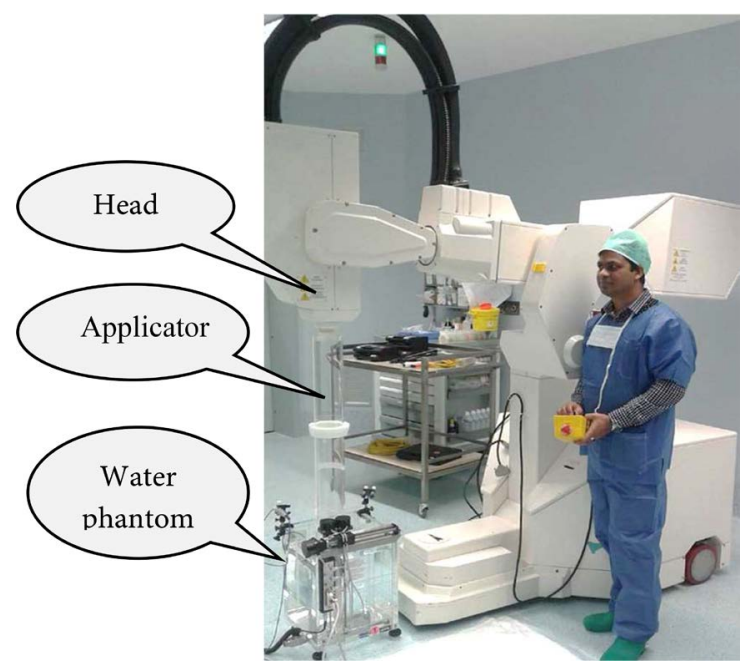

Figure 1. A photograph of the 3D moveable NOVAC7 system for IntraOperative radiation Therapy (IORT). 
B_ $(5 \mathrm{MeV}), \mathrm{C}_{-}(7 \mathrm{MeV})$ and $\mathrm{D}_{-}(9 \mathrm{MeV})$ by the manufacturer. For output measuring only two higher energies $\mathrm{C}_{-}(7 \mathrm{MeV})$ and $\mathrm{D}_{-}(9 \mathrm{MeV})$ were used. At the lowest energies A and B, the PDD curve around -Zmax is rather sharp and the uncertainty on the detector positioning at -Zmax has a pronounced effect on the detector response. This effect is much less important at the higher energies $\mathrm{C}$ and $\mathrm{D}$. And also, we cannot measure the output for less than $6 \mathrm{MeV}$ energy using microDiamond as the range of use energy of this detector for electron $6-25 \mathrm{MeV}$ [13]. The beam outputs were collimated through cylindrical applicators made of Perspex (PMMA) that fastened to the radiating head. In this work, applicators with inner diameters of $100 \mathrm{~mm}, 80 \mathrm{~mm}, 70 \mathrm{~mm}, 60 \mathrm{~mm}, 50 \mathrm{~mm}$ and $40 \mathrm{~mm}$ were used. The distance from source-to-surface (SSD) was $80 \mathrm{~cm}$, except for the applicator with the diameter of $100 \mathrm{~mm}$ for which the SSD was $100 \mathrm{~cm}$. For the dosimetric characterization of the beams percentage depth dose curves (PDD) were measured in a water phantom by means of a PTW 60017 Diode E detector. The dose per pulse at the depth of maximum dose was determined using alanine dosimeters.

\subsection{Dosimetry Systems and Electrometers}

The outputs measurements were performed for two energies and different applicators field size with PTW 60019 microDiamond and a PTW 60017 Diode E detectors, all by PTW (Physikalisch Technische Werkstaetten) Freiburg, Germany. The microDiamond is a waterproof and comparatively new synthetic single crystal diamond detector (SCDD). The features and advantages of this detector are excellent radiation hardness, temperature independence, near tissue-equivalence, synthetic production and consequently a high reproducibility of the dosimetric properties and good availability. Working principle of a high purity detector is, incoming ionizing radiation can push electrons from the valence band to higher energy levels thereby first filling electron traps caused by impurities and then bringing electrons to the conductivity band. A stable current can only be measured though after sufficient pre-irradiation to fill the traps. Contrariwise, the diode $\mathrm{E}$ is an unshielded $\mathrm{p}$-type silicon diode detector and is well established for dose distribution measurement in small and large fields electron beam. The characteristics and basic differences between the two detectors are shown in Table 1.

The microDiamond detector was positioned with its axis parallel to the beam direction as recommended by the manufacturer. The reference point was assumed to be positioned on the central axis of the device, $1 \mathrm{~mm}$ below the detector surface. The diode $\mathrm{E}$ detector was irradiated with its main axis parallel to the beam axis and its effective point of measurement was assumed $0.6 \mathrm{~mm}$ below the top surface following the manufacturer's. A PTW Unidos E Universal Dosemeter was used for the relative output factors determination.

\subsection{Dosimetric Measurements}

Measurements were performed in an MP3-XS IORT PTW Motorized water 
Table 1. Detectors and their characteristics.

\begin{tabular}{ccc}
\hline Type of Detector & $\begin{array}{c}\text { PTW 60019 } \\
\text { microDimond }\end{array}$ & $\begin{array}{c}\text { PTW 60017 } \\
\text { Diode E }\end{array}$ \\
\hline Type of product & SSCD & p-type silicon diode \\
Sensitive area $\left(\mathrm{mm}^{2}\right)$ & 3.80 & 1 \\
Thickness of volume $(\mu \mathrm{m})$ & 1 & 1 \\
Nominal Sensitive volume $\left(\mathrm{mm}^{3}\right)$ & 0.004 & 0.03 \\
Total window area density $\left(\mathrm{mg}^{2} \mathrm{~cm}^{2}\right)$ & 101 & 140 \\
Cover material & $0.3 \mathrm{~mm} \mathrm{RW3}$ & $0.3 \mathrm{~mm} \mathrm{RW3}$ \\
& $0.6 \mathrm{~mm}$ epoxy & $0.4 \mathrm{~mm}$ epoxy \\
Nominal response & $0.1 \mathrm{~mm} \mathrm{Al}$ & \\
Signal polarity & $1 \mathrm{nC} / \mathrm{Gy}$ & $9 \mathrm{nC} / \mathrm{Gy}$ \\
\hline
\end{tabular}

Abbreviation: SSCD, synthetic single crystal diamond; RW3, Goettingen White Water; Al, Aluminum; nC, nano-Coulomb; Gy, Gray

phantom. The PMMA applicators to define the field size were always positioned in vertical orientation, in contact with the water surface. To measure the output factor at various energies, both microDiamond and Diode detectors were placed inside the water phantom and irradiated at the depth of maximum dose for each combination of energy/applicator size. Dose readings were taken by PTW Unidos E Universal dosemeter for each field of interest and calculated as the average of three consecutive readings after delivering $300 \mathrm{MU}$. The output factor is calculated as the ratio of dose at Zmax for a given applicator to that of the reference applicator at the same energy, as in Equation (1).

$$
O F(E, d)=\frac{D_{w}\left(E, d, Z_{\max }\right)}{D_{w}\left(E, d_{r e f}, Z_{\max }\right)}
$$

where $D_{w}\left(E, d, Z_{\max }\right)$ is the calculated absorbed dose at depth $Z_{\max }$ in water for the beam collimated by the applicator with diameter $\mathrm{d}$ and $D_{w}\left(E, d_{\text {ref }} Z_{\max }\right)$ is the absorbed dose to water calculated for the reference field size (applicator diameter $10 \mathrm{~cm}$ ).

\section{Results and Discussion}

\subsection{Beam Characteristics}

The Percentage Depth Dose (PDD) curves obtained for the reference $(10 \mathrm{~cm})$ applicator with $\mathrm{A}_{-}(3 \mathrm{MeV}), \mathrm{B}_{-}(5 \mathrm{MeV}), \mathrm{C}_{-}(7 \mathrm{MeV})$ and $\mathrm{D}_{-}(9 \mathrm{MeV})$ energies by means of the PTW E 60017 diode are shown in Figure 2. The obtained PDD showed no significant change with applicator diameter $10 \mathrm{~cm}$, except for a slight increase of the percentage dose and some uncertainties in the surface region for lowest energy $\mathrm{A}_{-}(3 \mathrm{MeV})$ and $\mathrm{B}_{-}(5 \mathrm{MeV})$. Since no significant shift in the depth of maximum dose was observed for each energy, OF measurements were made at the same depth for all applicators, for example $1.1 \mathrm{~cm}$ and $1.2 \mathrm{~cm}$ for $\mathrm{C}_{-}$ $(7 \mathrm{MeV})$ and $\mathrm{D}_{-}(9 \mathrm{MeV})$, respectively. Table 2 shows the beam quality indices 


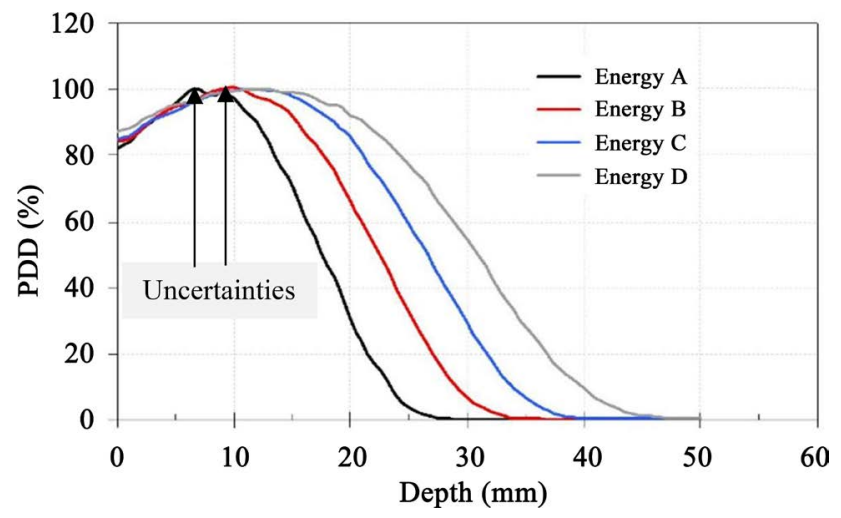

Figure 2. Percentage depth dose curves of for four energies for applicator with the diameter $10 \mathrm{~cm}$.

Table 2. Beam quality indices with $10 \mathrm{~cm}$ applicator.

\begin{tabular}{ccccc}
\hline Energy & $\mathbf{R}_{\mathbf{1 0 0}}(\mathrm{mm})$ & $\mathbf{R}_{\mathbf{8 0}}(\mathrm{mm})$ & $\mathbf{R}_{\mathbf{5 0}}(\mathrm{mm})$ & $\mathbf{R}_{\mathbf{3 0}}(\mathrm{mm})$ \\
\hline A & 7.0 & 13.5 & 17.6 & 4.10 \\
B & 10.0 & 17.7 & 22.4 & 5.22 \\
C & 11.0 & 21.1 & 26.5 & 6.18 \\
D & 12.0 & 24.2 & 30.8 & 7.18 \\
\hline
\end{tabular}

that obtained from the PDD curves with the $10 \mathrm{~cm}$ applicator. The dose per pulse measured by alanine dosimeters ranged from 29 to $63 \mathrm{mGy}$ for the $7 \mathrm{MeV}$ beams

\subsection{Output Factor}

Output factors were determined only for two highest energies codes $\mathrm{C}_{-}(7 \mathrm{MeV})$ and $D_{-}(9 \mathrm{MeV})$. The output factor values for different combinations of electron energy/applicator size determined by PTW 60019 microDiamond and PTW 60017 Diode E detectors are shown in Figure 3 and Figure 4 respectively.

From the above figures, it is shown that by increasing the field size at the same energy for both detectors, output factors are decreasing. By increasing the field size, the energy fluence within the radiation field decreases. Therefore, the absorbed dose and output factor decreases. On the other hand, by increasing the electron energy at the same field size, output factor increases. Increment of beam energy can increase the energy fluence. This increase in energy fluence would increment the output factor. Regarding output factor measurements, the microDiamond results were in good agreement with the diode $\mathrm{E}$ detector. But for this detector the results indicate a slight lower response than Diode E.

The mean relative difference between the output factors measured by two detectors at different energies and applicators size are listed in Table 3.

It is shown in Table 3, there is a good correlation between the results of output factors for both detectors. The maximum difference between the results of microDiamond and Diode detectors was equal to $2.74 \%$ and $2.17 \%$ in case of 40 mm applicator for $7 \mathrm{MeV}$ and $9 \mathrm{MeV}$ respectively. 


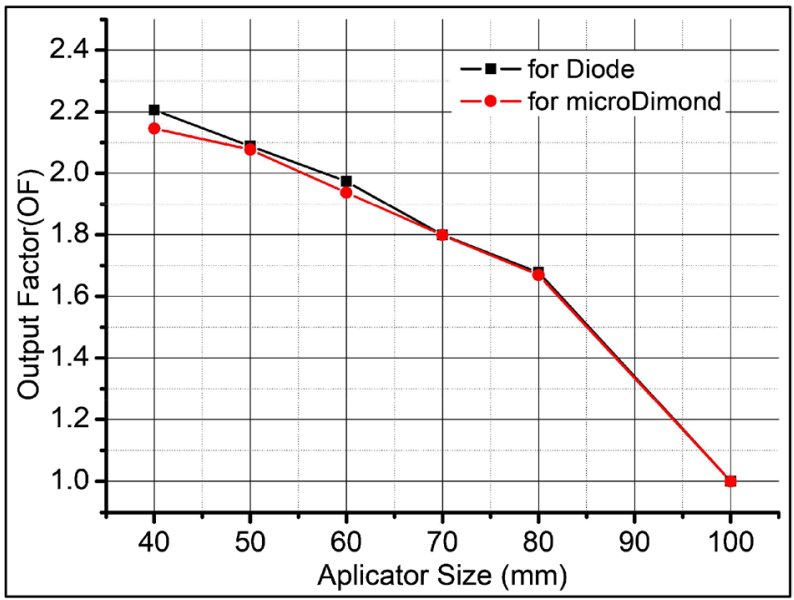

Figure 3. Output factor values for the energy $\mathrm{C}_{-}(7 \mathrm{MeV})$ with different applicator sizes measured by Diode and microDimond detectors.

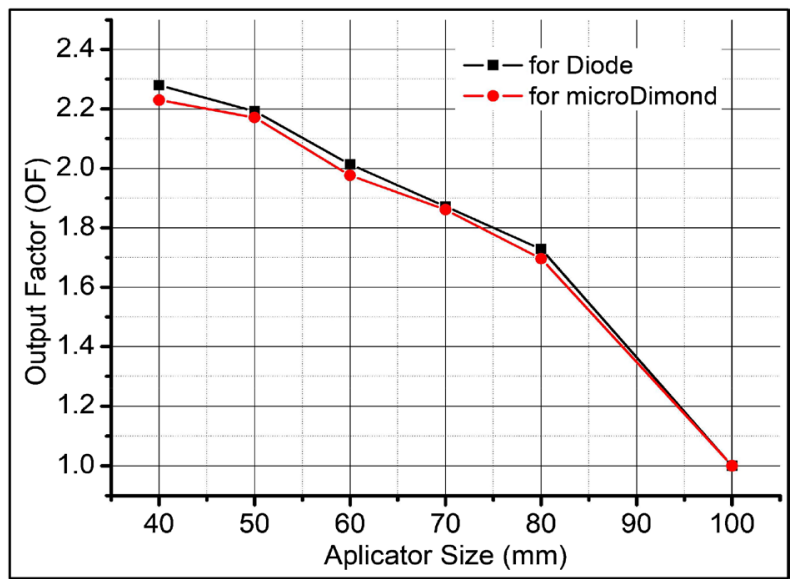

Figure 4. Output factor values for the energy $\mathrm{D}_{-}(9 \mathrm{MeV})$ with different applicator sizes measured by Diode and microDimond detectors.

Table 3. Relative differences of output factors for the energies with applicator size.

\begin{tabular}{ccc}
\hline \multirow{2}{*}{ Applicator Size $(\mathrm{mm})$} & \multicolumn{2}{c}{ Differences (\%) } \\
\cline { 2 - 3 } & Energy C_(7 MeV) & Energy D_(9 MeV) \\
\hline 40 & 2.74 & 2.17 \\
50 & 0.53 & 0.99 \\
60 & 1.86 & 1.86 \\
70 & 0.01 & 0.59 \\
80 & 0.50 & 1.09 \\
\hline
\end{tabular}

Many studies have been done during our work for measuring relative dosimetry as well as the output factors of IORT using microDiamond detector. Several authors published the OFs results as a comparison analysis of microDiamond with other detectors. In a study performed by Falco M. et al., [14] they showed for the silicon diode, an overestimation of the OF with respect to both the microDiamond and Advanced Markus ionization chamber (AM-IC) was observed 
in all fields below $6 \mathrm{~cm}$ in diameter for all the investigated energies. Maximum difference of $4.5 \%$ was found with respect to the AM-IC with the $6 \mathrm{MeV}$ beam using the $3 \mathrm{~cm}$ applicator. In another study, they investigated the capability of 5 types different detectors including microDiamond with Alamine dosimeters as reference for NOVAC7 in our Hospital. But they used different serial number microDiamond and other 4 detectors that are different from my used detectors [15]. They obtained the deviations from the reference OFs were generally within $1.5 \%$ for all the detectors.

The results obtained by two different detectors shown that there is no significant difference between the methods. So, the microDiamond detector is intended for use in measurements involving ionizing radiation and particularly in all situations where a high spatial resolution is to be achieved. The above agreements between the results shows, the microDiamond defector could be considered as a useful and reliable tool for relative dosimetry measurement in small field intraoperative electron beam.

\section{Conclusions}

One of the main aims of our study was to investigate and compare the dosimetric characteristics of a synthetic microDiamond detector with a solid state detector for electron beam. The synthetic PTW 60019 microDiamond detector was shown to exhibit excellent properties for output factors measurements in comparison with well-established PTW 60017 Diode E detector in small fields of electron beam. Indeed, for this detector, the results indicate a slight lower-response with dose per pulse. Differences in output factors measured by the microDiamond and the PTW 60017 Diode E detector were found 2.17\%, with the largest differences in high-energy/small field conditions.

The microDiamond dosimetric characteristic OF, makes this dosimeter suitable for precise dosimetry in Intra Operative Radiation Therapy with high-dose-per-pulse electron beams. Compared to the ionization chamber, the microDiamond does not require any correction for influence quantities, charge recombination and stopping power variation with water depth. Based on the results and other features like allowing excellent spatial resolution for very precise beam, it may be concluded that the PTW 60019 microDiamond could be considered as an accurate tool for output factors as well as relative dosimetry measurement of intraoperative electron beam.

\section{Acknowledgements}

This work was supported by the IAEA-ICTP/UniTS, a joint Master of Medical Physics programme under funding by the International Atomic Energy Commission (IAEA). M Rafiqul Islam wishes to thank ASST Papa Giovanni XXIII Hospital for providing technical and materials support. The authors wish to thank Cyclotron and Radioisotope Center, Tohoku University and GO PROTON JAPAN Inc as part of this work was carried out under the Cooperative 
Research Project Program between them.

\section{Author Disclosure}

M Rafiqul Islam: PhD Research Student, Tohoku University, JAPAN, was a Medical Physics Resident at ASST Papa Giovanni XXIII Hospital in Bergamo, ITALY, and is a permanent employ of BAEC, Bangladesh.

\section{Conflicts of Interest}

The authors declare no conflicts of interest regarding the publication of this paper.

\section{References}

[1] Krengli, M., Calvo, F.A., Sedlmayer, F., Sole, C.V., Fastner, G., Alessandro, M., Maluta, S., Corvò, R., Sperk, E., Litoborski, M., Pisani, C., Fillini, C., Fusconi, F., et al. (2013) Clinical and Technical Characteristics of Intraoperative Radiotherapy. Strahlentherapie und Onkologie, 189, 729-737. https://doi.org/10.1007/s00066-013-0395-1

[2] Hensley, F.W. (2017) Present State and Issues in IORT Physics. Radiation Oncology, 12, 37. https://doi.org/10.1186/s13014-016-0754-z

[3] ICRU I. (2008) Report 80: Dosimetry Systems for Use in Radiation Processing. Journal of ICRU, 8, 29-70. https://doi.org/10.1093/jicru/ndn027

[4] Almond, P.R., Biggs, P.J., Coursey, B.M., Hanson, W.F., Huq, M.S., Nath, R. and Rogers, D.W.O. (1999) AAPM's TG-51 Protocol for Clinical Reference Dosimetry of High-Energy Photon and Electron Beams. Medical Physics, 26, 1847-1870. https://doi.org/10.1118/1.598691

[5] Laitano, R.F., Guerra, A.S., Pimpinella, M., Caporali, C. and Petrucci, A. (2006) Charge Collection Efficiency in Ionization Chambers Ex-Posed to Electron Beams with High Dose per Pulse. Physics in Medicine \& Biology, 51, 6419-6436. https://doi.org/10.1088/0031-9155/51/24/009

[6] Saini, A.S. and Zhu, T.C. (2004) Dose Rate and SDD Dependence of Commercially Available Diode Detectors. Medical Physics, 31, 914-924. https://doi.org/10.1118/1.1650563

[7] Di Venanzio, C., Marinelli, M., Milani, E., Prestopino, G., Verona, C., Verona-Rinati, G., Falco, M., Bagal, P., Sanoti, R. and Pimpinella, M. (2013) Characterization of a Synthetic Single Crystal Diamond Schottky Diode for Radiotherapy Electron Beam Dosimetry. Medical Physics, 40, Article ID: 021712. https://doi.org/10.1118/1.4774360

[8] Bagalà, P., Di Venanzio, C., Falco, M.D., Guerra, A.S., Marinelli, M., Milani, E., Pimpinella, M., Pompili, F., Prestopino, G., Santoni, R., Tonnetti, A., Verona, C. and Verona-Rinati, G. (2013) Radiotherapy Electron Beams Collimated by Small Tubular Applicators: Characterization by Silicon and Diamond Diodes. Physics in Medicine \& Biology, 58, 8121-8132. https://doi.org/10.1088/0031-9155/58/22/8121

[9] De Angelis, C., Soriani, A., Benassi, M. and Onori, S. (2006) On Measuring the Output of an IORT Mobile Dedicated Accelerator. Radiation Protection Dosimetry, 120, 221-225. https://doi.org/10.1093/rpd/nci621

[10] Björk, P., Knöös, T. and Nilsson, P. (2004) Measurements of Output Factors with Different Detector Types and Monte Carlo Calculations of Stopping-Power Ratios 
for Degraded Electron Beams. Physics in Medicine \& Biology, 49, 4493-4506. https://doi.org/10.1088/0031-9155/49/19/004

[11] Griessbach, I., Lapp, M., Bohsung, J., Gademann, G. and Harder, D. (2005) Dosimetric Characteristics of a New Unshielded Silicon Diode and Its Application in Clinical Photon and Electron Beams. Medical Physics, 32, 3750-3754. https://doi.org/10.1118/1.2124547

[12] Güngör, G., Aydın, G., Mustafayev, T.Z. and Özyar, E. (2019) Output Factors of Ionization Chambers and Solid State Detectors for Mobile Intraoperative Radiotherapy (IORT) Accelerator Electron Beams. Journal of Applied Clinical Medical Physics, 20, 13-23. https://doi.org/10.1002/acm2.12522

[13] User Manual for PTW Micro-Diamond Type 60019. https://www.ptwdosimetry.com/en/products/microdiamond

[14] Falco, M., Marinelli, M., Tonnetti, A., Rinati, G.V., Pimpinella, M., Ciccotelli, A., De Stefano, S., Felici, G. and Marangoni, F. (2015) Characterization of High-Dose-per-Pulse Intraoperative Radiation Therapy Electron Beams by Using a Micro-Diamond Dosimeter. Physica Medica, 31, 897-902. https://doi.org/10.1016/j.ejmp.2015.06.008

[15] Pimpinella, M., Andreoli, S., De Angelis, C., Della Monaca, S., D’Arienzo, M. and Menegotti, L. (2019) Output Factor Measurement in High Dose-per-Pulse IORT Electron Beams. Physica Medica, 61, 94-102.

https://doi.org/10.1016/j.ejmp.2019.04.021 